Man bringt die zu untersuchende Chromatlösung in ein Becherglas oder einen Kolben, verdünnt, wenn nöthig, mit Wasser bis auf mindestens $50 c c$ und versetzt hierauf mit Ammoniak, oder mit Salz- oder Schwefelsäure bis zur sehr schwach sauren Reaction.

Das zur Titration benutzte Wasserstoffsuperoxyd wird stark verdünnt. Man fügt zweckmässig zu dem käuflichen Wasserstoffsuperoxyd das 5 -, 10- oder 20 fache Volumen an reinem Wasser; in dieser Verdünnung hält es sich bei gewöhnlicher Temperatur längere Zeit unverändert.

Die Wasserstoffsuperoxydlösung setzt man aus einer Glashahnbürette der Chromatlösung zù, welche man hierbei über ein weisses Papier hält. Die ersten Tropfen erzengen in der gelben Flüssigkeit dunkel gefärbte Flecken, die bald verschwinden; nach und nach nimmt die ganze Flüssigkeit eine gleichmässig blaue Farbe an, welche bei dem Umschwenken jedoch wieder verschwindet, so dass zuletzt, je nach der Menge des vorhandenen Chroms, die Flüssigkeit mehr oder minder intensiv grün gefärbt. erscheint. Die Titration ist beendet, sobald der letzte Tropfen keinen blauen Fleck mehr hervorruft.

Die Wasserstoffsuperoxydlösung wird auf eine Kaliumbichromatlösung von bekanntem Gehalt unter denselben Bedingungen gestellt.

Um keine zu stark gefärbten Flüssigkeiten zu erhalten, darf man nicht mehr als $0,2-0,3 g$ Chromsäure anwenden, da sonst die vorübergehende Blaufärbung nur schwierig zu erkennen ist.

Ueber Anwendung des Wasserstoffsuperoxyds bei der Bestimmung des Chroms, des Mangans und des Eisens macht A. Carnot*) Mittheilungen.

1. Ch rom. Während die Chromsäure in kalter und saurer Lösung durch Wasserstoffsuperoxyd rasch und vollständig reducirt wird, vollzieht sich in warmer alkalischer Lösung oder bei Gegenwart von Ammoniak der umgekehrte Process, das Chromoxyd wird vollständig in Chromsäure übergeführt. Diese Oxydation des Chromoxyds mit Wasserstoffsuperoxyd empfiehlt sich namentlich aus dem Grunde, weil hierbei das Zufügen irgend welcher fixer Reagentien vermieden werden kann.

Fügt man zu einer verdünnten und auf $100^{\circ} \mathrm{C}$. erhitzten Lösung ron Chromchlorid einige Cubikcentimeter Wasserstoffsuperoxyd, übersättigt hierauf mit Ammoniak und erhitzt wieder zum Kochen, so ent-

*) Bulletin de la société chimique de Paris [3. sér.] 1, 277. 
steht zunächst eine braune Trübung, welche jedoch bald verschwindet and in eine klare, gelbe Lösung übergeht, indem sich chromsaures Ammon bildet. Die Reaction wird durch Umschütteln des Kolbens beschleunigt. Sollte etwas Chromoxyd der Oxydation entgangen sein, so lässt man dasselbe absitzen, decantirt die Lösung und wiederholt, nachdem das Chromoxyd durch Zusatz von Säure wieder in Lösung übergeführt worden ist, das oben geschilderte Verfahren.

Mittelst desselben kann man das Chrom vollständig, wenn man die Oxydation in ammoniakalischer Lösung wiederholt, von den durch Ammon und kohlensaures Ammon fällbaren Oxyden trennen. Im Filtrate wird nach schwachem Ansäuern mittelst Wasserstoffsuperoxyds die Chromsäure reducirt und nun die Flüssigkeit zum Kochen erhitzt. Würde jetzt direct das Chromoxyd mit Ammoniak gefällt, so müsste, im Falle noch ein Rest von unzersetztem Wasserstoffsuperoxyd vorhanden wäre, wieder eine theilweise Oxydation des Chroms stattfinden, und wäre die Fällung hierdurch eine unvollständige. Um diesen möglichen Fehler sicher zu umgehen, leitet der Verfasser, bevor er das Chromoxyd mit Ammoniak fällt, in die erwärmte Lösung ganz kurz einen Strom von Schwefelwasserstoffgas ein.

Zur Bestimmung des Chroms kann man nach Carnot*) auch die schwach saure, mit Wasserstoffsuperoxyd reducirte Lösung mit phosphorsaurem und hierauf mit essigsaurem Natron oder Ammon versetzen und alsdann 10-15 Minuten zum Kochen erhitzen. Da die Flüssigkeit hierbei sauer bleibt, so ist jede Wiederoxydation ausgeschlossen, und das Chrom scheidet sich vollständig als grünes Phosphat ab.

2. Mangan. Ebenso wie bei dem Chrom wirkt Wasserstoffsuperoxyd auch bei dem Mangan in saurer Lösung reducirend und in alkalischer Lösung oxydirend. Die erste Reaction ist wohl bekannt und wird zur Bestimmung des Wasserstoffsuperoxyds mittelst Permanganatlösung benutzt. Auch die zweite Reaction verdient besondere Erwähnung, da sich dieselbe nach $\mathrm{Carnot}$ zur Bestimmung des Mangans sehr gut eignet.

Versetzt man die Lösung eines Mangansalzes mit einigen Cubikcentimetern Wasserstoffsuperoxyd und darauf mit Ammoniak, so scheidet sich sämmtliches Mangan in Form eines dunkelbraunen Niederschlags ab. Sobald man die Lösung zum Kochen erhitzt, setzt sich derselbe

*) Vergl. diese Zeitschrift 22, 244. 
$a b$ und lässt sich nun leicht filtriren. In den Niederschlag gèt jedoch stets ein Theil der Basen mit über, die in der Lösung enthalten sind; von diesen ist er nur durch wiederholte, in gleicher Weise auszuführende Fällungen zu befreien. Anstatt den so erhaltenen Niederschlag auf gewichtsanalytischem Wege zu bestimmen, lässt sich auch ein titrimetrisches Verfahren vortheilhaft zur Anwendung bringen. Carnot hat nachgewiesen, dass der auf diesem Wege erzeugte Manganniederschlag sich immer im Zustande derselben Oxydation befindet, wie er durch die Formel $\mathrm{Mn}_{6} \mathrm{O}_{11}$ oder $5 \mathrm{MnO}_{2}$, $\mathrm{MnO}$ dargestellt wird, und dies sowohl, wenn das Mangan allein vorhanden, als auch, wenn es in Gegenwart von Kalk, Baryt, Zinkoxyd und Eisenoxyd gefällt. worden ist. Man hat also nur die ammoniakalisch gemachte Lösung 10 Minuten zu kochen, um das überschüssige Wasserstoffsuperoxyd vollständig zu zersetzen, und alsdann in dem Niederschlage den disponiblen Sauerstoff volumetrisch, z. B. mit Oxalsäure, Schwefelsäure und Kaliumpermanganat, zu bestimmen. 5 Aequivalente disponibler Sauerstoff entsprechen, wie sich aus obiger Formel ergibt, genau 6 Aequivalenten Mangan.

3. Eisen. In Betreff des Eisens erwähnt Carnot die bekannte Thatsache, dass sich das Eisen im Gegensatz zu den beiden vorher besprochenen Metallen, durch Wasserstofisuperoxyd in saurer Lösung oxydiren lässt, und zwar vollzieht sich die Oxydation sofort und in der Kälte.

\section{Zur Bestimmung des Chroms and des Kupfers in Eisen und Stahl unter Anwendung von Natriumhypophosphit als Reductionsmittel.} C. Reinhardt*) empfiehlt das Natriumhypophosphit als Reductionsmittel, welches Fisenchlorid bei dem Erhitzen selbst in concentrirter Lösung und bei starkem Säureüberschuss in verhältnissmässig kurzer Zeit reducirt und den Vortheil bietet, dass es Schwefelwasserstoff nicht zersetzt, was vor allem hinsichtlich eines vorhandenen Ueberschusses in Betracht kommt. Das Salz ist hygroskopisch, und verwendet es der Verfasser daher in wässeriger Lösung. ${ }^{*}$ )

*) Stahl und Eisen 9, 404.

**) $200 \mathrm{~g}$ Natriumhypophosphit werden bei gewöhnlicher Temperatur in $400 c c$ Wasser gelöst; die sich ergebende Lösung wird nach einige Tage langem: Stehen filtrirt. 